\title{
Conception and Implementation of a Supermarket Shopping Assistant System
}

\author{
Antonio Marin-Hernandez, Guillermo de Jesús Hoyos-Rivera, Marlon García-Arroyo, \\ Luis Felipe Marin-Urias \\ Department of Artificial Intelligence, \\ Universidad Veracruzana, \\ Xalapa, Mexico \\ anmarin@uv.mx,ghoyosr@gmail.com,morfeos_ms@hotmail.com,luisfelipe.marin@ieee.org
}

\begin{abstract}
In this paper we present the design framework of a shopping assistant system to be used in supermarkets mainly by elderly or disabled people. The whole system is based on the interaction of three different kinds of electronic devices: a) mobile devices that users carry with them (smart phones or electronic tablets), b) autonomous mobile robots assisting users by displaying information and carrying groceries; and finally, c) the supermarket technological infrastructure (database servers, Wi-Fi and Bluetooth access points, etc.). These components interact through a common technological platform, allowing the user to prepare his/her shopping list, and then using it with the robot as an assistant, during his/her shopping process.
\end{abstract}

Keywords-Human-Robot Interaction, Service Robots, Ambient Intelligence, Intelligent Distributed Systems

\section{INTRODUCTION}

Over last years robots have evolved from manufacturing cells, to museum guides, passing successful commercial applications, such as vacuum cleaners, lawn movers and pool cleaners. As both, robots' hardware and software became more complex, new applications, particularly to serve humans in a closer way, are projected. Service robots should have the ability of move and interact with their environment, but mainly with their users, common people, in a friendly way.

On the other hand, while e-commerce has been increasing over the last years, due mainly to the possibility of getting better prices, many people still prefer to visit stores. This it is mainly because people's shopping decision is frequently not only guided by the price of the products, but other factors such as the ambiance of shopping, professional consultation, seeing, touching and trying the products, etc., may have an important effect [1].

In this order of ideas Service Robots are an interesting solution for helping people on proposed supermarket environments, as they can, apart of carrying groceries, provide useful information such as prices, stock, products location, etc.

In this paper we present the design and experimental implementation of a prototype system to be used in supermarkets, conceived primarily for helping people, possibly with mobility limitations, such as elderly or physical disabled, while doing their shopping.

As an elementary overview of the operation of our proposal, we can say that the user uses a mobile device in order to prepare the shopping list. Then, upon arriving to the supermarket, the user identify himself/herself with the supermarket's shopping technological infrastructure (server), transmitting at this point the shopping list. Then, once the user identified, it is assigned an assistant robot for keep him/her company while shopping. Based on the user's choice, and in order to improve the overall performance of the system, the supermarket server may store some information about the user, such as credit card information, user preferences, shopping habits, etc.), so that the supermarket may "learn" about its users, and using this information for improving the shopping experience.

This paper is organized as follow, in section 2, a review of some of the more important related work is presented. In section 3 we present the proposed design and the main parts of our proposal are described in detail. Section 4 concerns the current implementation as well as the evaluation of our proposal made by potential users. Finally in section 5 we present our conclusions and future work.

\section{RELATED WORKS}

To improve the quality of the services provided to users in supermarkets many developments have been proposed, some of them are nowadays successful applications currently used in different establishments, all around the world. These proposals and developments can be roughly divided in: local infrastructure and fixed locations (kiosks), mobile agents running on personal devices and mobile robotics platforms.

\section{A. Local Infrastructure and Fixed Locations}

The most common use of electronic devices in supermarkets has been, for a while, the interactive kiosks, developed for helping people to locate products or services. For example, there are applications on kiosks that allow users to choose a wine and/or a recipe in terms of the selected food.

One of the first proposals including a semiautonomous 
process is VeggieVision [2] which consist on a scale system able to distinguish different products with the use of a variety of features like, color, texture and density. Recent improvements of this technology [3], allow us to have in supermarkets, scales systems capable of automatically detect the fruits and vegetables that are weighted. Even if these scales are not still completely robust, they reduce the intervention of the users, to select in some cases, between two or three similar instances [4].

These kiosks are commonly fixed and spread over the supermarket, making customers to search and move to them if they require information.

\section{B. Personal Devices}

The use of personal devices (hand-held) improves usersystem interaction while allowing high mobility. Many approaches have been proposed on these devices. For example in [5] it has been proposed the use of a Personal Shopping Assistance that personalizes attention based on individual needs. The system is based on mobile devices and a centralized local server that communicate in real-time.

In [6] an augmented reality shopping assistant device, called PromoPad, is proposed. This hand-held device is able to place products in a contextual setting and can provide complimentary products, enhancing the shopping experience by displaying products and useful information on the screen.

The problem of human interaction with hand-held devices has been treated in [1]. In order to have a friendly interaction they propose the use of an humanized interface.

\section{Service Robots}

While personal devices offer mobility, recent studies, as in [7], show that elderly people prefer a conversational robot than a quiet companion assistance. These studies have been achieved with a partially tele-operated robot in real conditions with users not familiarized at all with robotics research.

Service robots in supermarket and/or mall environments present many challenging tasks, some of them have been solved at least partially. For example in [8], autonomous mobile robot localization (SLAM) and reactive path planning problems are addressed. The problem of customer detection and path planning as an anticipatory behavior for a friendly social interaction has been treated on [9] and [10].

In [11] it is presented a shopping assistance robot for visually impaired. In this work, the robot is able to provide user with spatial cognition and information about products selection. On other hand, telepresence through the use of robots in supermarkets has also been tried, for example in [12], where a robot for remote shopping is presented. This robot is able to manipulate objects on this environments based on a teleoperated approach.

Autonomous robot as shopping guides is another explored branch in the field, for example in [13] it is presented a robot that can interact with people on a supermarket environment and guide them to a location where some products are located. The robot stays for a while waiting for scanning products or if necessary to call a salesperson. If the customer does not require more assistance the robot begins a new exploration in the search of new customers. On the mentioned work, the robot does not accompany the client during their shopping and only offers, some punctual services, as mentioned.

In order to have a friendly human-robot interaction, robots require showing "emotions" as a human affective retro-alimentation process; these problems have been treated in [14] and [15].

\section{SySTEM DESCRIPTION}

In this paper, we propose the design of a complete shopping assistance system that permits people, possibly elderly of disabled, to have the support of an physical agent during all their shopping process with the added value of a complete connectivity with supermarket resources and a friendly user-interface. Although our proposal is addressed for any kind of public, elderly or disabled people may be considered a particularly important target, since this kind of developments might help them in accomplishing their shopping with a reduced effort.

Our proposal is composed of three main active components: smartphones, mobile robots, and local infrastructure. In the Fig. 1, it is shown the general architectural structure of the proposed system.

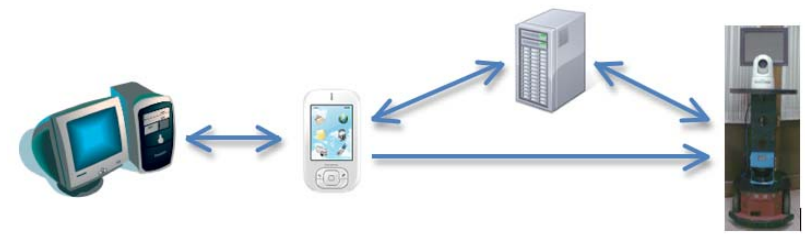

Figure 1. Schema of the proposed system communication

As we can clearly see, in this figure the main components of our proposal appear. The user can upload his/her shopping list, either directly or by using a laptop or desktop computer, to a mobile device. Then, and without detailing at this moment technical aspects, when arriving at the shopping center, the user's shopping list is uploaded to the store server, and once a robot has been assigned to the user, the user's shopping list is sent to the robot, and the interaction between the robot and the user can then start. This mode to work is the main subject of this work, and will be explained in detail in the next sections. 


\section{A. Scenario description}

Entering into a more detailed description, and assuming that the user has already captured his/her shopping list into the mobile device, the system interaction begins with the arriving of user to the supermarket main entrance. At that moment the user sets a personal mobile device to establish communication with supermarket sever in order to provide user identification and consequently user's shopping list.

The user is then asked to identify himself by a Personal Identification Number (PIN) that has been previously stored in the system. Once done, the local system assigns a personal service robot that will go to meet the client. A new ID confirmation is done at this stage in order to assure a successful contact with assigned robot.

Knowing people preferences personal robot is able to provide the best path to follow at the interior of supermarket in order to accomplish the filling of the products noted on the user's list. At all moment, client could take another path to search for another product not in list or to take a look on some of the available offers. Robot is then able to provide at all moment new paths in function of the user actions.

In case that the user prefers following his own path, robot does not provide any proposal and then follows customer at all moment. Robot is able at all moment to establish communication with local servers through the wireless connection (Wi-Fi or Bluetooth) and can recover useful information about products such as expiration date, number of units in stock, etc.

In order to ensure an effective service, the system should also be responsible for maintaining the shopping list, for example by marking taken products and adding products not initially listed, but added by the user.

\section{B. Mobile Devices}

When going shopping most people prepare a shopping list on a piece of paper. Then, as long as the client goes picking the products on the list, and eventually, some others write a mark on their list, denoting that a given product has already been put in the shopping cart. This rudimentary way to work is the usual way for the majority of people while doing shopping, and it has been like this since a long time ago.

Today, the use of mobile devices as mobile phones has become an essential part in the life of many people mainly in urban areas. Therefore, the use of smartphones or pads for creating shopping lists, instead of the traditional piece of paper, sounds to be a logical evolution.

Moreover, given the fact that this kind of devices have already built-in communication facilities, it would not be absurd thinking about the possibility of transmitting a shopping list to the system. Then it could be capable to help users in a more effective way.

The user's mobile device is primarily used as a medium for capturing and transporting the shopping list and as a way of identification. However, this device could be also a way of communication between the user and the robot or the supermarket, for example, when user and robot separated caused by a crowd.

\section{The Supermarket Shopping Server}

An application running on the Supermarket Shopping Server, will manage the connection requests coming from the users' mobile devices. When a new user is registered in the supermarket, the server adds the MAC address of the mobile device to the list of known devices and associating this address with the user's ID. Once this is done, the server receives the shopping list and selects an idle Robotic Assistant, providing it with the user's shopping list, and assigning it to the user. The user is informed about which Robotic Assistant has been assigned to him/her.

This server will be in charge of managing and coordinating the whole operation of the system, from the initial connection, to the transmission of the shopping lists from the mobile devices, and to the robots, as well as of managing all the logistics related to the administration of the robots themselves.

At any moment the server will be able to detect the position into the supermarket of every single robot, determining also, the percentage of the shopping list already in the shopping cart (which would be a medium for estimating the time left for the user into the supermarket), as well as the level of charge of the battery of each robot, and in this way establishing the best strategy for sending them to be recharged.

Crossing the information about the status of the robot, as well as the shopping habits of a particular client (let us say, how much time that client takes for doing his shopping), the server will be capable to do the best assigning of robots to users.

This is not a simple task, and should be carefully performed in order to allow every single robot to fully satisfy the expectations of every single user.

\section{The Robotic Assistant}

Apart of proposing a plausibly optimized path and/or to follow a client while he/she is picking products, the robot will display all the time on its screen the user's shopping list and the total cost of products currently carried.

A user wishing to add a given product to the shopping cart should present it to the robot. Then the robot would present on its screen the relevant information about the product itself, asking the user whether or no to add it to the shopping cart and how many units. In this way the robot would keep a record of the shopping activity of the user. On the screen of the robot it would be permanently displayed the shopping list, clearly indicating which products are already in the shopping cart, and which of them are not yet. 
On the other hand, if the item is not in the list, the Robotic Assistant will ask the user whether he/she wants to add it or not. Also if the user shows an item that is already checked, the Robotic Assistant will ask if more units of that item should be added, eliminated or totally removed.

\section{CurRent Implementation}

In order to validate our system design and before testing on real conditions, a local implementation has been done.

\section{A. Mobile Devices}

We have implement an application for mobile devices using the Java 2 Micro Edition (J2ME) on a mobile phone. This application allows to edit the shopping list directly on the device, or receiving a list previously edited on a laptop or desktop computer.

On Fig. 2, it is shown the current design of this application. It is very important to note that no restrictions about the way of writing the list are given to the user. The user can write in his/her list, in the same way as it would do it on a piece of paper, e.g. kind of products (milk, sugar, soap, etc.), specific brands or generic names. The way as it can be discriminated and matched by the system is an actual field of research of the group and it is out of the scope of this paper.

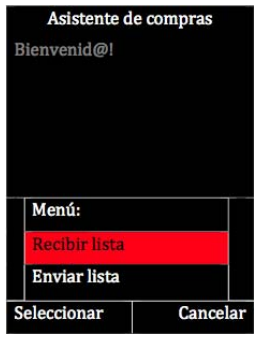

(a)

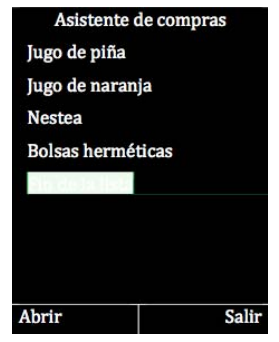

(b)

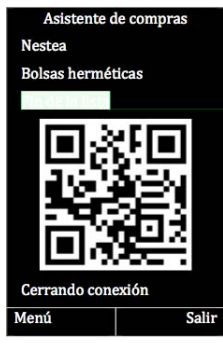

(c)
Figure 2. Application developed for a mobile device. a) Initial state of the app, and b) example of a list written by a user, and c) Identification QR-code.

As previously stated, when a user enters in the store, his/her mobile device application communicates with the supermarket servers providing user's ID and shopping list. The supermarket server asks then for an ID confirmation by the previously mentioned PIN, avoiding with that identity theft. Once the ID verified, supermarket servers sends to the mobile device an QR-code which should be used at this stage of the project as identification with the assigned robot.

\section{B. Local Server and Infrastructure Communication}

The server at this stage is a desktop computer connected to the local network, providing devices communication by
Wi-Fi and Bluetooh access points in the local building.

Once server has corroborated user's identity and send it to him/her the QR-code, it contact the robot and send the user's shopping list, the information contained in the QR-code and some users preferences. At this stage only if user prefer to follow or being followed by the robot are considered as preferences.

\section{Mobile Robotic Assistant}

The Robotic Assistant prototype was implemented using a mobile robot platform Pioneer 3DX from Adept Mobile Robots with a laser range finder, a complete ring of sonars and bumpers. In order to fulfill project requirements, a pan/tilt/zoom IP camera, a touch screen, microphone and speakers have been attached. This mobile platform is able to carry a small supermarket caddy for groceries.

Our mobile robot platform has already implemented different modules for navigation and localization, path planning, face recognition, and some other, not described in this paper. A specific module for supermarket Human-Robot interaction has been developed for this project, taking into account previously mentioned considerations.

In order to have a friendly human-robot interaction, the display of the robot has been divided in three zones: a) a region where is shown the user's shopping list and their evolution, b) a zone where is provided information, for example at the beginning here is shown the stored photo of the user, and c) a region where the user can see what the robot's camera is detecting (Fig 3).

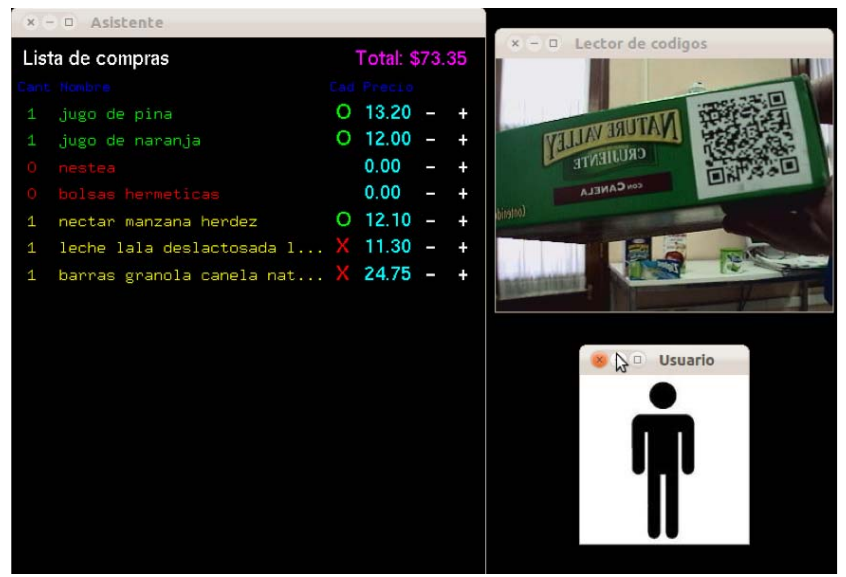

Figure 3. Human-robot interface running on robot. Display is divided in three regions, a zone with the current shopping list, the current image taken by the robot and useful information that could interest user.

In order to help the robot obtaining more of the product information, in an automatic way, such as expiration date, price, calories, a brief description, etc. It is necessary to implement a different and more efficient method than a 
simple barcode detection, which only provides an id of the product. Having most of the information in the product itself will reduce queries and network traffic, as also it will accelerates processes. This is the reason why we have decided to set 2D QR-codes into the products, the robot is able to detect and recognize this codes and obtain all the information stored on it.

On the other hand, humans do not like to deal with kiosk to search for product prices. In fact in [4] it is reported that sometimes users prefer to leave products that are not properly labeled before dealing with kiosks. To take this into account, it is essential that the robotic assistance has the ability to track products in front of the camera. In order to deal with this aspect, a fuzzy controller over pan, tilt and zoom camera capabilities has been implemented [16], making easier QR-code detection and recognition, while reducing human effort.

Once an QR-code has been detected and decoded, the robot searches for the product in the users shopping list: if the product is on it then product is colored on green (initially all products are in red), if the product is not in the list the product is adde to it and displayed in a yellow color. User are able to see evolution of their list as they are picking products, and at the same time they could have an estimation of the total account.

Application has also the possibility of adding or eliminating products by $\mathrm{a}+$ or - signs aside the product in the list (Fig. 3). In future implementations this will be done automatically, by detecting products in the caddy.

\section{Users Evaluation}

In order to obtain a feedback on how this system can be introduced to the society, as well as the easiness on using our entire framework, we have performed some user studies in the street with people which are not related with robotics. We took 51 random participants aged between 17 and 73 years old, those people were invited to try the system and then to answer some questions about their experience.

It is important to mention that in the study we made with real users we did not focus specially on elderly or disabled people since a representative sample was not at our reach. So we took a sample of simply non-specialist potential users, and them assuming that the obtained results can be assumed for every kind of user.

The information, obtained from the survey, is divided in two: the evaluation of the easiness of use and the people's opinion about the possibility of implementing a system like ours in a. In the first part the participants have shown a good acceptance of this kind of technology as it is illustrated in Figure 4, the preferences were rated on one hand in easiness/usefulness from 1, "not friendly at all", to 5 "very easy to use". On the other hand in their aspect, 1 for "not understandable/ugly" to 5 "very clear".

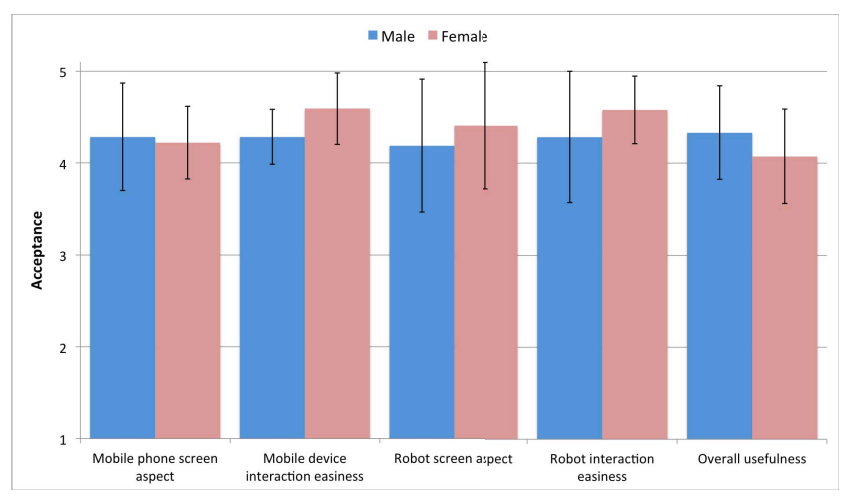

Figure 4. Users evaluation on the easiness of use and aspect of the different parts of the framework.

In the second part, we wanted to study if it would be viable to have this robot as a supermarket service or as other service (i.e. personal robot or anything else), and we could obtain some curious information about this particular service robot as we can observe on Figure 5, people show a markable preference in having this application as a service provided by the supermarket rather than a personal robot, it may be caused by costs of the product. Nevertheless, male users show to be more interested on personal robots than female users, so the market publicity for personal robots at home may be more oriented to men than to women.

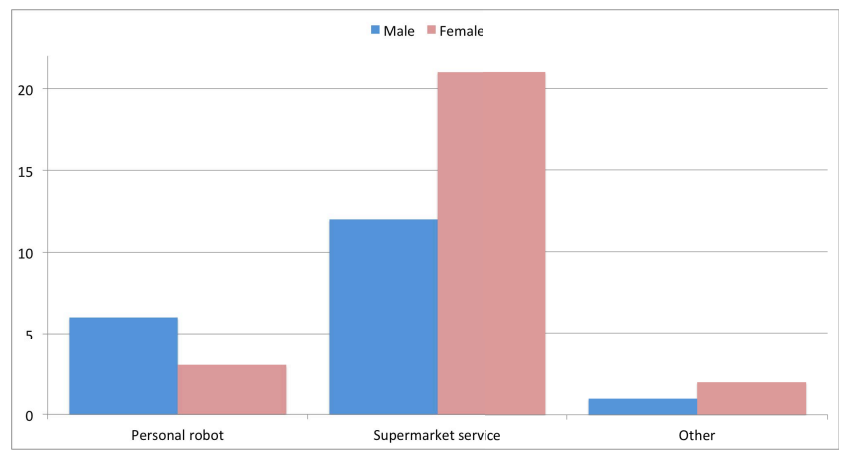

Figure 5. Users preferences on where this robot application must be introduced to the society.

Based on these studies we could infer that the system can be exploited in different ways: help for handicapped, company for elderly, information retrieval for supermarkets as for users and market strategies for inviting custumers, among other applications.

\section{CONCLusions AND Future WORK}

In this paper it has been presented a new framework for supermarket shopping assistance robots, which it is intended to help not only to handicapped people in doing shopping 
with more freedom, but also to regular people, providing instant information about products, the total amount of the shopping as also company for people, between other applications.

As far as we can see this is a very relevant work, since we have not found similar works when analyzing the state of the art. This project is only the beginning of a whole system, which can support and aid elderly and handicapped people. We tried to create a system that could be used by anyone, with a simple interface and bringing the product information that the user could need. Other modules like manipulation, navigation or speech will be incorporated to the system with the participation and collaboration of students and professors. In future implementations a face recognition module is considered to be developed and implemented.

In some of the implementations where we are currently working, the server will search each item of the users list on the store database, showing which products are not in stock in advance, allowing the user to decide if he/she wants or not to buy that day. Furthermore, we will obtain more information about human preferences, measures and opinions are needed in order to get a more robust, complete and acceptable service robot system.

\section{REFERENCES}

[1] M.-Y. S. . J.-S. C. . C.-J. Hu, "Design of a shopping assistance agent system," Information Technology Journal, vol. 10, no. 6, pp. $1186-1193,2011$.

[2] R. Bolle, J. Connell, N. Haas, R. Mohan, and G. Taubin, "Veggievision: a produce recognition system," in Applications of Computer Vision, 1996. WACV '96., Proceedings 3rd IEEE Workshop on, dec 1996, pp. $244-251$.

[3] A. Rocha, D. C. Hauagge, J. Wainer, and S. Goldenstein, "Automatic fruit and vegetable classification from images," Computers and Electronics in Agriculture, vol. 70, no. 1, pp. 96 - 104, 2010.

[4] K. Kalyanam, R. Lal, and G. Wolfram, "Future store technologies and their impact on grocery retailing," in Retailing in the 21st Century, M. Krafft and M. K. Mantrala, Eds. Springer Berlin Heidelberg, 2006, pp. 95-112.

[5] A. Asthana, M. Cravatts, and P. Krzyzanowski, "An indoor wireless system for personalized shopping assistance," in Mobile Computing Systems and Applications, 1994. Proceedings., Workshop on, dec 1994, pp. $69-74$.

[6] C. B. Wei, Z.; Owen, "Design of the promopad: An automated augmented-reality shopping assistant," Journal of Organizational and End User Computing, vol. 20, pp. 41 -56, 2008.
[7] A. M. Sabelli, T. Kanda, and N. Hagita, "A conversational robot in an elderly care center: an ethnographic study," in Proceedings of the 6th international conference on Humanrobot interaction, ser. HRI '11. New York, NY, USA: ACM, 2011, pp. 37-44.

[8] H.-M. Gross, A. Koenig, H.-J. Boehme, and C. Schroeter, "Vision-based monte carlo self-localization for a mobile service robot acting as shopping assistant in a home store," in Intelligent Robots and Systems, 2002. IEEE/RSJ International Conference on, vol. 1, 2002, pp. 256 - 262 vol.1.

[9] B. Johansson and C. Balkenius, "An experimental study of anticipation in simple robot navigation," in Anticipatory Behavior in Adaptive Learning Systems, ser. Lecture Notes in Computer Science, M. Butz, O. Sigaud, G. Pezzulo, and G. Baldassarre, Eds. Springer Berlin / Heidelberg, 2007, vol. 4520, pp. 365-378.

[10] S. Satake, T. Kanda, D. F. Glas, M. Imai, H. Ishiguro, and N. Hagita, "How to approach humans?: strategies for social robots to initiate interaction," in Proceedings of the 4th ACM/IEEE international conference on Human robot interaction, ser. HRI '09. New York, NY, USA: ACM, 2009, pp. 109-116.

[11] C. Gharpure and V. Kulyukin, "Robot-assisted shopping for the blind: issues in spatial cognition and product selection," Intelligent Service Robotics, vol. 1, pp. 237-251, 2008.

[12] T. Tomizawa, K. Ohba, A. Ohya, and S. Yuta, "Remote food shopping robot system in a supermarket -realization of the shopping task from remote places," in Mechatronics and Automation, 2007. ICMA 2007. International Conference on, aug. 2007, pp. $1771-1776$.

[13] H.-M. Gross, H. Boehme, C. Schroeter, S. Mueller, A. Koenig, E. Einhorn, C. Martin, M. Merten, and A. Bley, "Toomas: Interactive shopping guide robots in everyday use - final implementation and experiences from long-term field trials," in Intelligent Robots and Systems, 2009. IROS 2009. IEEE/RSJ International Conference on, oct. 2009, pp. 2005 -2012 .

[14] T. Kanda, M. Shiomi, Z. Miyashita, H. Ishiguro, and N. Hagita, "An affective guide robot in a shopping mall," in Proceedings of the 4th ACM/IEEE international conference on Human robot interaction, ser. HRI '09. New York, NY, USA: ACM, 2009, pp. 173-180.

[15] M. Shiomi, T. Kanda, D. Glas, S. Satake, H. Ishiguro, and N. Hagita, "Field trial of networked social robots in a shopping mall," in Intelligent Robots and Systems, 2009. IROS 2009. IEEE/RSJ International Conference on, oct. 2009, pp. $2846-2853$.

[16] A. Perez-Garcia, V. Ayala-Ramirez, and R. E. Sanchez-Yanez, "Geno-fuzzy design of a visual servoing system," Telecommunications and Radio Engineering, vol. 64, no. 7-12, pp. 865-872, 2005. 